\title{
GOLDENHAR SYNDROME IN ASSOCIATION WITH PROFOUND MENTAL RETARDATION
}

Suresh R${ }^{1}$, Sudha Rudrappa², Nirmala V. Pinto ${ }^{3}$

\section{HOW TO CITE THIS ARTICLE:}

Suresh R, Sudha Rudrappa, Nirmala V. Pinto. "Goldenhar Syndrome in Association with Profound Mental Retardation". Journal of Evolution of Medical and Dental Sciences 2014; Vol. 3, Issue 44, September 15;

Page: 10877-10880, DOI: $10.14260 /$ jemds/2014/3419

ABSTRACT: Goldenhar syndrome is a variant of hemifacial microsomia. It is not usually associated with mental retardation; even if present it is mild to moderate. In this case both profound mental retardation and bilateral facial microsomia are present. Early identification and intervention can improve the intellectual functioning, otherwise favorable prognosis.

INTRODUCTION: In 1952 Maurice Goldenhar described a variant of hemifacial microsomia called Goldenhar syndrome. It results from an aberrant development of the $1^{\text {st }}$ and $2^{\text {nd }}$ branchial arches. It has an incidence of approximately 1 in 5600, more common among males with a ratio of 2:1 and also common among whites.[1] Goldenhar syndrome is a form of occulo - auriculo - vertebral spectrum (OAVS), as a minimal sign microtia must be present. ${ }^{[2]}$ It presents bilaterally in $10-33 \%$ of cases. ${ }^{[3]}$ About $10 \%$ of children may have mental retardation, usually mild to moderate. ${ }^{[4]}$ Here we report a case of Goldenhar syndrome with profound mental retardation which is very unusual.

CASE REPORT: A 1yr 5month old male child who was first born to 3rd degree consanguineously married couple, presented to hospital with history of fever, cough, hurried breathing, chest in drawing of two days duration. There was no history suggestive of cyanosis, recurrent pneumonia or hospital admissions in the past.

There was no significant family history of major medical or genetic disorders. During the antenatal period, the mother underwent regular antenatal check-ups with only one antenatal scan done in the third trimester, which was told to be normal. This baby was delivered at term through caesarean section at our institution with a birth weight of $3 \mathrm{~kg}$ without any significant neonatal events.

There was a history of developmental delay since birth with development age corresponds to 7-8months at the time of presentation. The child was sick looking with significant respiratory distress. He was euglycemic, afebrile, maintained saturation in room air with normal heart rate. Head to toe examination of the child revealed auricular abnormalities like microtia with pre-auricular tag bilaterally and ocular abnormalities like micro-opthalmous, micro-cornea, iris-coloboma and retinochoroidal coloboma bilaterally, child also had hypoplastic mandible as illustrated in figure 1 .

There was no facial asymmetry or cranial nerve involvement, no other obvious anatomical abnormality was present. Child had extensive crepitations on both the sides, other systems were normal. Child had a silent precordium with single first and second heart sound without any murmur. Liver span was $6 \mathrm{~cm}$ along the mid-clavicular line, there were no focal neurological deficits. An initial working diagnosis of lower respiratory tract infection probably bronchopneumonia with syndromic facies was made. 
On evaluation, counts were elevated with neutrophil predominance, blood culture had no growth, and chest x-ray had features of bronchopneumonia. Hearing assessment revealed moderate conductive hearing loss on both the sides.

Two dimensional echocardiography and colour Doppler showed congenital heart disease patent ductus arteriosus(PDA) of size $5 \mathrm{~mm}$ with left to right shunt, pressure gradient of $61 \mathrm{mmHg}$ with dilated left atrium and left ventricle. I.Q assessment revealed profound mental retardation.

The above clinical and evaluatory findings were suggestive of Goldenhar syndrome with bronchopneumonia. The baby was treated with intravenous antibiotics for seven days and other supportive care was given. Child was advised hearing aid and ocular corrective surgery.

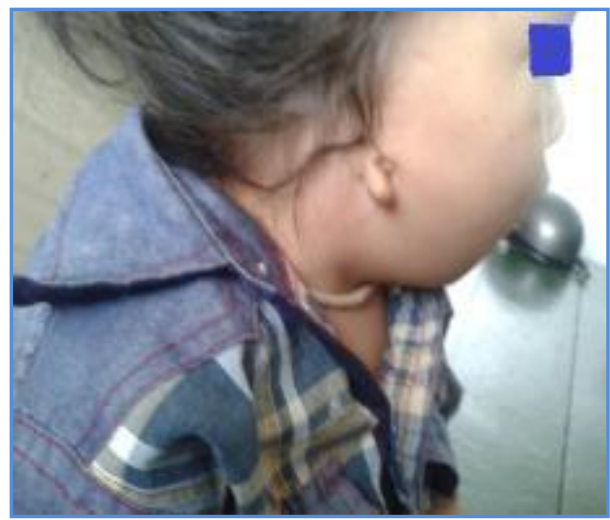

Figure 1

Microtia with preauricular tag

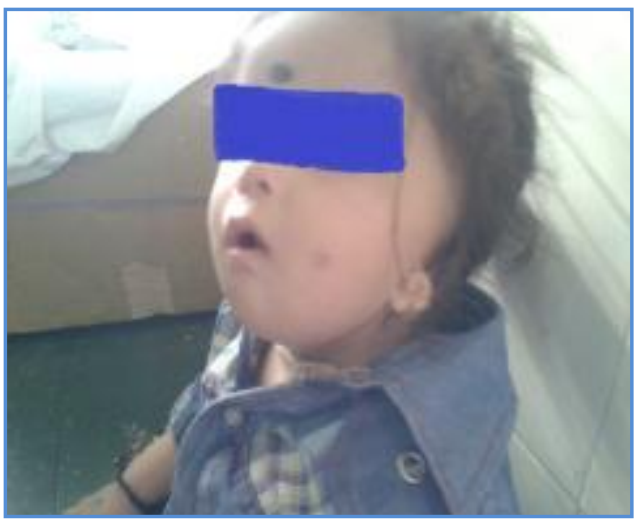

Figure 2

Hypoplastic Mandible.

DISCUSSION: Goldenhar described a syndrome with epibulbar dermoids, pre-auricular appendages and mandibular hypoplasia (hemifacial microsomia) which was expanded by Gorlin et.al to "occuloauriculovertebral spectrum". Most cases are sporadic but some familial cases also occur, they have been associated with a deletion on the long arm of chromosome 22(22q11.2).

This syndrome has variable expression ranging from preauricular tags to cleft lip. The term "Extended Goldenhar complex" includes the involvement of cardiac, renal, pulmonary and central nervous system including severe hydrocephalus and mental retardation. ${ }^{[5]}$ Incidence is 1 in 3000 5000 live births. Males are more often affected. If one sibling is affected the risk of recurrence is $3 \% .6]$

There are two theories which explains the etiology,

1. Disruption of the vascular supply to first and second branchial arches at about 30-40days of gestation.

2. Disruption of the neuronal crest cell migration and its distribution.

1. CLINICAL FEATURES: Craniofacial - Marked facial asymmetry is seen in $20 \%$ of cases whereas mild facial asymmetry is seen in $65 \%$ of cases, about 10-30 percentage of patients will have bilateral involvement. Aplasia or hypoplasia of mandible can occur and sometimes occur in association with microstomia, cleft lip; cleft palate and parotid gland agenesis are also known to 
occur. b. Ocular - Epibulbar dermoids occur in 35\% of cases, narrow palpebral fissure occurs in $10 \%$ of cases, micro-opthalmia and colobomas are seen in several patients with this syndrome. Auricular - Agenesis or hypoplasia of both external and middle ear can occur, both conductive and sensory neural hearing loss can occur, although occurrence of sensory neural hearing loss is rare $(10 \%)$.

- Cardiovascular system - Ventricular septal defect and tetrology of fallot constitute half of the cardiac anomalies and no single characteristic cardiac lesion can be attributed to this syndrome.

- Respiratory system - Ranges from incomplete lobulation to agenesis. Tracheo-esophageal fistula has also been documented.

- Gastro-intestinal system - Imperforate anus with or without rectovaginal fistula can occur.

- Renal - Renal anomalies include agenesis, double ureter, hydronephrosis, hydroureter and reno-vascular abnormalities.

- Central nervous system - This includes skull defects like plagiocephaly, cranial nerve weakness especially facial nerve and brain malformations. Mental retardation is present in only $5-15 \%$ of the cases with mild-moderate severity.

- Musculo-skeletal - This includes cervical vertebral fusion defects, radial limb anomalies and talipes equino varus deformity.

2. EVALUATION: An extensive diagnostic evaluation is required. Craniofacial evaluation includes imaging studies to determine the extent of middle ear involvement and auditory evoked responses for assessment of hearing loss. Skeletal radiological survey for cranial, vertebral and limb anomalies. Echocardiography to detect septal defects, renal sonography to evaluate the urinary tract anatomy. Ophthalmology, otolaryngology and nutritional evaluations are often needed. Molecular studies are indicated in patients with severe and extensive anomalies, several chromosomal abnormalities are associated with this syndrome like del5p, del6q, del8q, del18q, del22q, trisomy 7,8 and 18 but a normal result can be expected for children with this syndrome.

3. MANAGEMENT: Surgical procedures are of major importance to correct life threatening abnormalities. Cosmetic and functional surgical management can involve excision of preauricular tags and transposition of the microtic ear. Surgical repair of mandible can also be done. Conductive hearing loss is often corrected by surgery and hearing aid requirement is very less. Closure of the cardiac septal defects is also feasible. Surgical management of bony deformities and ocular abnormalities can be done.

4. PROGNOSIS: Prognosis depends on the severity of the phenotype and is poor if there is associated intellectual disability. In general prognosis is usually favourable.

5. ANTENATAL DIAGNOSIS: It is mainly done by screening pregnant women. The earliest diagnosis can be made at 16 weeks of gestation based on maxillary clefts and micropthalmia.

CONCLUSION: Goldenhar syndrome if associated with mental retardation, the prognosis is poor. Although surgical corrective procedures are available for most deformities, a proper prenatal evaluation can prevent the worse outcome of many genetic syndromes including Goldenhar syndrome. 


\section{REFERENCES:}

1. Lorie M Harper, Alison G cahil. Goldenhar syndrome. Obstetric Imaging. Elsevier. Philadelphia. 2012. pp 600-601.

2. Helga V Toriello, Shelly D Smith. Genetic hearing loss with external ear abnormalities. Heriditary hearing loss and its syndromes. $3^{\text {rd }}$ ed, Oxford University Press, USA. 2013. pp 221.

3. Dirk Kunet, Hennie Kramer. Inherited Syndromic hearing Impairment. Genetics for ENT Specialits, Remedica, London 2005, pp 87-90.

4. Michael C Broadsky. Pediatric neuro-opthalmology. Syndromes with Neuro-opthalmologic overlap. $2^{\text {nd }}$ ed, Springer, London.2010, pp 573-574.

5. Michael Entezami, Matthias Albig, Adam Gasiorek-Wiens, Rolf Becker. Ultrasound diagnosis of foetal anomalies. Thieme, Germany. 2004 pp 248.

6. Jones KL. Oculo-auriculo-Vertebral spectrum (Goldenhar). In smith's recognizable patterns of human malformation. $6^{\text {th }}$ ed. Elsevier. Philadelphia 2006. pp 738.

\section{AUTHORS:}

1. Suresh R.

2. Sudha Rudrappa

3. Nirmala V. Pinto

\section{PARTICULARS OF CONTRIBUTORS:}

1. Post Graduate Student, Department of Paediatrics, Mysore Medical College and Research Institute, Mysore.

2. Professor, Department of Paediatrics, Mysore Medical College and Research Institute, Mysore.

3. Post Graduate Student, Department of Paediatrics, Mysore Medical College and Research Institute, Mysore.
NAME ADDRESS EMAIL ID OF THE CORRESPONDING AUTHOR:

Dr. Sudha Rudrappa,

Professor \& Head, Department of Paediatrics, Mysore Medical College and Research Institute, Mysore. Email: sudharudrappa1@gmail.com

Date of Submission: 02/09/2014. Date of Peer Review: 03/09/2014. Date of Acceptance: 09/09/2014. Date of Publishing: 15/09/2014. 